\title{
Universal programmable devices for unambiguous discrimination
}

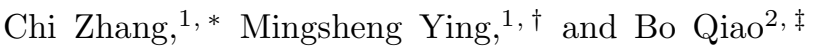 \\ ${ }^{1}$ State Key Laboratory of Intelligent Technology and Systems, \\ Department of Computer Science and Technology Tsinghua University, Beijing, China, 100084 \\ ${ }^{2}$ Department of Fundamental Science, Tsinghua University, Beijing, China, 100084
}

(Dated: July 31, 2018)

\begin{abstract}
We discuss the problem of designing unambiguous programmable discriminators for any $n$ unknown quantum states in an $m$-dimensional Hilbert space. The discriminator is a fixed measurement which has two kinds of input registers: the program registers and the data register. The quantum state in the data register is what users want to identify, which is confirmed to be among the $n$ states in program registers. The task of the discriminator is to tell the users which state stored in the program registers is equivalent to that in the data register. First, we give a necessary and sufficient condition for judging an unambiguous programmable discriminator. Then, if $m=n$, we present an optimal unambiguous programmable discriminator for them, in the sense of maximizing the worst-case probability of success. Finally, we propose a universal unambiguous programmable discriminator for arbitrary $n$ quantum states.
\end{abstract}

PACS numbers:

\section{INTRODUCTION}

Discrimination between quantum states is an essential task in quantum communication protocols. Generally, a set of states cannot be discriminated exactly, unless they are orthogonal to each other [1]. One strategy of discriminating non-orthogonal quantum states is the so-called unambiguous discrimination: with a non-zero possibility of getting inconclusive answer, one can distinguish the given states without error [2, 3, 4, [5, 6, 7, 8, 9, 10]. Such a strategy works if and only if the states to be distinguished are linearly independent [6] , and finding the optimal unambiguous discrimination through Bayesian approach with a given priori probability distribution, can be reduced to a semi-definite programming (SDP) problem [7, 8]. On the other hand, D'Ariano et al [9] considered the problem of finding optimal unambiguous discrimination through "minimax strategy". In such a strategy, no information about priori probability is needed, and the discriminator is designed to maximize the smallest of the success probabilities.

All above discriminators depend on the set of states being discriminated. When states change, the device also needs to be changed. Recently, the problem of designing programmable discriminator attracted a lot of attention. In a programmable quantum device, quantum states are input through two kinds of registers: program registers and data registers. The states in data registers are manipulated by the fixed device, according to the states in program registers 11, 12, 13, 14, 15, 16, 17. Particularly, in a programmable discriminator, the information about states being discriminated is offered through a "quantum program", according to which, the discrimination on the

\footnotetext{
*Electronic address: zangcy00@mails.tsinghua.edu.cn

${ }^{\dagger}$ Electronic address: yingmsh@tsinghua.edu.cn

${ }^{\ddagger}$ Electronic address: qiaobo`2268@163.com
}

state in data register is specified. Different from the discriminators for known states, a programmable discriminator is capable to discriminate any states, with the corresponding program. In Ref. [18], Dušek et al provided a model of unambiguous programmable discriminator for a pair of 1-qubit states. In this model, a new quantum state, besides the pair of states being discriminated, is needed for programming. Recently, Bergou et al 19] constructed an alternative unambiguous programmable discriminator for any two different states. The advantage of this discriminator is that, the "quantum program" is simply comprised of the states being discriminated. Furthermore, unambiguous programmable discriminator for two states with a certain number of copies is also discussed 21, 22]. All of above tasks focus on discriminating two states, and estimates the efficiency with a given priori probability. In addition, Fiurášek et al 20. considered several kinds of programmable quantum measurement devices, including a device performing von Neumann measurement on a qudit, which can also be regarded as a discriminator for $d$ orthogonal states.

In this paper, we describe the more general unambiguous programmable discriminators for any $n$ quantum states. The quantum program used in these discriminators is the tensor product of the $n$ states being discriminated, so that there is no extra states needed for programming. We design the optimal discriminators in a minimax strategy, in order to avoid any dependence on the priori information. Since quantum states can be unambiguously discriminated if and only if they are linearly independent, we strict our discussion under this condition, and claim a programmable discriminator "universal" if it can unambiguously discriminate any set of linearly independent states.

Our present article is organized as follows. Section. 【 is a preliminary section in which we recall some results needed in the sequel from linear algebra 23]. In section. IIII we give a necessary and sufficient condition for unambiguous programmable discriminators. Further, in 
section. IV we define the efficiency of a discriminator under the minimax strategy, and provide a set of properties for the optimal discriminators. Then, we present the optimal unambiguous programmable discriminators for $n$ arbitrary quantum states in an $n$-dimensional Hilbert space in section. $\nabla$ and propose a set of unambiguous programmable discriminators for $n$ quantum states in an $m$-dimensional Hilbert space, where $m>n$, in section. VI In section. VII we conclude the paper with a short summary.

\section{PRELIMINARIES}

Let us begin with some preliminaries that are useful in presenting our main results.

The antisymmetric tensor product of states $\left|\varphi_{1}\right\rangle,\left|\varphi_{2}\right\rangle$, $\cdots,\left|\varphi_{n}\right\rangle$ in a Hilbert space $H$ is defined as

$$
\begin{aligned}
& \left|\varphi_{1}\right\rangle \wedge\left|\varphi_{2}\right\rangle \wedge \cdots \wedge\left|\varphi_{n}\right\rangle \\
= & \frac{1}{\sqrt{n !}} \sum_{\sigma \in S(n)} \operatorname{sgn}(\sigma)\left|\varphi_{\sigma_{1}}\right\rangle\left|\varphi_{\sigma_{2}}\right\rangle \cdots\left|\varphi_{\sigma_{n}}\right\rangle,
\end{aligned}
$$

where $S(n)$ is the symmetric (or permutation) group of degree $n, \operatorname{sgn}(\sigma)$ denotes the signature of permutation $\sigma$, i.e., $\operatorname{sgn}(\sigma)=+1$, if $\sigma$ is an even permutation; $\operatorname{sgn}(\sigma)=-1$, if $\sigma$ is an odd permutation. The span of all antisymmetric tensors $\left|\varphi_{1}\right\rangle \wedge\left|\varphi_{2}\right\rangle \wedge \cdots \wedge\left|\varphi_{n}\right\rangle$ in $H^{\otimes n}$ is denoted by $\wedge^{n} H$, called the antisymmetric subspace of $H^{\otimes n}$. If the dimension of $H$ is $m$, then the dimension of $\wedge^{n} H$ is $\left(\begin{array}{c}m \\ n\end{array}\right)$.

In an $n$-composite system, for any $\sigma \in S(n)$, we also use $\sigma$ to represent a linear operation on the system, which realigns the subsystems according to $\sigma$, i.e.,

$$
\sigma\left|\varphi_{1}\right\rangle\left|\varphi_{2}\right\rangle \cdots\left|\varphi_{n}\right\rangle=\left|\varphi_{\sigma_{1}}\right\rangle\left|\varphi_{\sigma_{2}}\right\rangle \cdots\left|\varphi_{\sigma_{n}}\right\rangle
$$

here $|\varphi\rangle=\left|\varphi_{1}\right\rangle\left|\varphi_{2}\right\rangle \cdots\left|\varphi_{n}\right\rangle$ is an arbitrary product state in the $n$-composite system. It is easy to prove that $\sigma$ is a unitary operation. For a state $|\psi\rangle \in H^{\otimes n},|\psi\rangle \in \wedge^{n} H$ if and only if for any $\sigma \in S(n)$,

$$
\sigma|\psi\rangle=\operatorname{sgn}(\sigma)|\psi\rangle
$$

In this paper, we denote the projector of $\wedge^{n} H$ by $\Phi(n)$. For any product state $|\varphi\rangle=\left|\varphi_{1}\right\rangle\left|\varphi_{2}\right\rangle \cdots\left|\varphi_{n}\right\rangle$ in $H^{\otimes n}$,

$$
\langle\varphi|\Phi(n)| \varphi\rangle=\frac{1}{n !} \operatorname{det}(X),
$$

where $X$ is the Gram matrix of $\left\{\left|\varphi_{1}\right\rangle,\left|\varphi_{2}\right\rangle, \cdots,\left|\varphi_{n}\right\rangle\right\}$, i.e., the $(i, j)$ element of $X$ is

$$
X_{(i, j)}=\left\langle\varphi_{i} \mid \varphi_{j}\right\rangle
$$

Hence, the Eq.(4) equals to zero if and only if $\left\{\left|\varphi_{1}\right\rangle,\left|\varphi_{2}\right\rangle, \cdots,\left|\varphi_{n}\right\rangle\right\}$ are linearly dependent.

\section{UNAMBIGUOUS PROGRAMMABLE DISCRIMINATOR}

An unambiguous programmable discriminator for $n$ quantum states in an $m$-dimensional Hilbert space $H$, can be simply designed in the following version. The discriminator has $n$ program registers and one data register. When the quantum state wanted to be identified is selected in states $\left|\psi_{1}\right\rangle, \ldots,\left|\psi_{n}\right\rangle$, the $i$ th program register is put in the state $\left|\psi_{i}\right\rangle$, for $i=1, \ldots, n$, and the data register is prepared in the state wanted to be identified. Here, we label the $i$ th program register as the $i$ th subsystem, the data register as the $(n+1)$ th subsystem, and use $\bar{i}$ to indicate the system consisting of all subsystems under consideration except the $i$ th one. For simplicity, we introduce a notation $\left|\alpha_{t}^{s}\right\rangle$ to denote a special kind of product states in a $(s+1)$-component quantum system, where the state in the $l$ th subsystem is $\left|\alpha_{l}\right\rangle$, for any $1 \leq l \leq s$, and the state in the $s+1$ subsystem is the same as the $t$ th subsystem, i.e.,

$$
\left|\alpha_{t}^{s}\right\rangle=\left|\alpha_{1}\right\rangle\left|\alpha_{2}\right\rangle \cdots\left|\alpha_{s}\right\rangle\left|\alpha_{t}\right\rangle
$$

Then, if the data register is in $\left|\psi_{j}\right\rangle$, the total input state is $\left|\psi_{j}^{n}\right\rangle=\left|\psi_{1}\right\rangle\left|\psi_{2}\right\rangle \cdots\left|\psi_{n}\right\rangle\left|\psi_{j}\right\rangle$. The discriminator is described by a general POVM $\left\{\Pi_{0}, \Pi_{1}, \cdots, \Pi_{n}\right\}$ on the entire input system, including all program registers and the data register. For any $i \neq j, i \neq 0$, if it is satisfied that $\left\langle\psi_{j}^{n}\left|\Pi_{i}\right| \psi_{j}^{n}\right\rangle=0$, then when outcome $i(i \neq 0)$ is observed, one may claim with certainty that the data register is originally prepared in the state $\left|\psi_{i}\right\rangle$, and occurrence of outcome 0 means that the identification fails to give a report. In this paper, we also use $\vec{\Pi}$ to denote the measurement $\left\{\Pi_{0}, \Pi_{1}, \cdots, \Pi_{n}\right\}$ for simplicity.

The main purpose of this section is to present a necessary and sufficient condition for unambiguous programmable discriminators. We would like to start with a lemma for positive operators, which will be useful in the proof for the necessary and sufficient condition.

Lemma 1 Suppose $\Omega$ is a positive operator on a composite system $A B$, for any product state $|\varphi\rangle=\left|\varphi_{a}\right\rangle_{A}\left|\varphi_{b}\right\rangle_{B}$, it holds that

$$
\langle\varphi|\Omega| \varphi\rangle \operatorname{Tr}(\Omega) \leq\left\langle\varphi_{a}\left|\operatorname{Tr}_{B}(\Omega)\right| \varphi_{a}\right\rangle\left\langle\varphi_{b}\left|\operatorname{Tr}_{A}(\Omega)\right| \varphi_{b}\right\rangle
$$

where $\operatorname{Tr}_{A}\left(\operatorname{Tr}_{B}\right)$ is the partial trace over the subsystem $A(B)$.

Proof. It is observed that $\Omega / \operatorname{Tr}(\Omega)$ satisfies the trace condition and positivity condition for a density operator. Let $\rho=\Omega / \operatorname{Tr}(\Omega)$, which can be regarded as a density operator, and consider a quantum operation $\varepsilon=\operatorname{Tr}_{B} \otimes$ $\operatorname{Tr}_{A}$. Then

$$
F(\varepsilon(\rho), \varepsilon(|\varphi\rangle\langle\varphi|)) \geq F(\rho,|\varphi\rangle\langle\varphi|),
$$

where $F$ stands for the fidelity between two density operators 1]. Because $|\varphi\rangle$ is a pure product state, we have 
that

$$
\begin{aligned}
& \langle\varphi|\Omega| \varphi\rangle \operatorname{Tr}(\Omega) \\
= & \langle\varphi|\rho| \varphi\rangle(\operatorname{Tr}(\Omega))^{2} \\
\leq & \left\langle\varphi_{a}\left|\operatorname{Tr}_{B}(\rho)\right| \varphi_{b}\right\rangle\left\langle\varphi_{b}\left|\operatorname{Tr}_{A}(\rho)\right| \varphi_{b}\right\rangle(\operatorname{Tr}(\Omega))^{2} \\
= & \left\langle\varphi_{a}\left|\operatorname{Tr}_{B}(\Omega)\right| \varphi_{a}\right\rangle\left\langle\varphi_{b}\left|\operatorname{Tr}_{A}(\Omega)\right| \varphi_{b}\right\rangle .
\end{aligned}
$$

This completes the proof.

Theorem 1 A measurement $\left\{\Pi_{0}, \Pi_{1}, \cdots, \Pi_{n}\right\}$ is an unambiguous programmable discriminator for any $n$ quantum states in Hilbert space $H$, if and only if the support space of $\operatorname{Tr}_{i}\left(\Pi_{i}\right)$ is a subspace of $\wedge^{n} H$, i.e.,

$$
\operatorname{supp}\left(\operatorname{Tr}_{i}\left(\Pi_{i}\right)\right) \leq \wedge^{n} H,
$$

where $\operatorname{Tr}_{i}$ is the partial trace over the ith subsystem, and $\wedge^{n} H$ is the antisymmetric subspace of $H^{\otimes n}$.

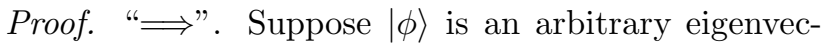
tor of $\Pi_{i}$ with non-zero eigenvalue, since $\Pi_{i}$ is a positive operator,

$$
\left\langle\psi_{j}^{n} \mid \phi\right\rangle=0,
$$

for any $j \neq i$. To prove Eq.(10), we only have to prove that

$$
\operatorname{supp}\left(\operatorname{Tr}_{i}(|\phi\rangle\langle\phi|)\right) \leq \wedge^{n} H .
$$

Let $\{|1\rangle,|2\rangle, \ldots,|m\rangle\}$ be an orthonormal basis for Hilbert space $H$. As $|\phi\rangle \in H^{\otimes(n+1)}$, it can be rewritten as

$$
|\phi\rangle=\sum_{\omega} v(\omega)|\omega\rangle
$$

where $|\omega\rangle$ is the orthonormal basis of space $H^{\otimes(n+1)}$, derived from the given basis of $H$, i.e.,

$$
|\omega\rangle=\left|\omega_{1}\right\rangle\left|\omega_{2}\right\rangle \cdots\left|\omega_{n+1}\right\rangle,
$$

where $\left|\omega_{k}\right\rangle \in\{|1\rangle,|2\rangle, \ldots,|m\rangle\}, 1 \leq k \leq n$, and $v(\omega)$ is the corresponding coefficient. Because Eq.(11) should be satisfied with any input states under consideration, we can choose some special states to derive necessary conditions for $|\phi\rangle$.

First, choose $\left|\psi_{j}\right\rangle=|s\rangle$, where $|s\rangle \in\{|1\rangle, \cdots,|m\rangle\}$,

$$
\begin{aligned}
\left\langle\psi_{j}^{n} \mid \phi\right\rangle & =\sum_{\omega} v(\omega)\left\langle s \mid \omega_{j}\right\rangle\left\langle s \mid \omega_{n+1}\right\rangle \prod_{k \neq j}\left\langle\psi_{k} \mid \omega_{k}\right\rangle \\
& =\sum_{\omega_{\bar{j}}} v\left(\omega \mid \omega_{j}=\omega_{n+1}=s, \omega_{\bar{j}}\right)\left\langle\psi_{\bar{j}} \mid \omega_{\bar{j}}\right\rangle,
\end{aligned}
$$

where

$$
\left|\psi_{\bar{j}}\right\rangle=\left|\psi_{1}\right\rangle\left|\psi_{2}\right\rangle \cdots\left|\psi_{j-1}\right\rangle\left|\psi_{j+1}\right\rangle \cdots\left|\psi_{n}\right\rangle,
$$

and

$$
\left|\omega_{\bar{j}}\right\rangle=\left|\omega_{1}\right\rangle\left|\omega_{2}\right\rangle \cdots\left|\omega_{j-1}\right\rangle\left|\omega_{j+1}\right\rangle \cdots\left|\omega_{n}\right\rangle .
$$

Since $\left|\psi_{\bar{j}}\right\rangle$ can be any product state in $H^{\otimes(n-1)}$ and $\left|\omega_{\bar{j}}\right\rangle \mathrm{s}$ form an orthonormal basis for $H^{\otimes(n-1)}$, to confirm that Eq. (15) always equals to zero, it must holds that, $v(\omega)=$ 0 , if $\omega_{j}=\omega_{n+1}$, for some $j \neq i$.

Next, choose $\left|\psi_{j}\right\rangle=\frac{1}{\sqrt{2}}(|s\rangle+|t\rangle)$, where $|s\rangle,|t\rangle \in$ $\{|1\rangle,|2\rangle, \ldots,|m\rangle\}$,

$$
\begin{aligned}
\left\langle\psi_{j}^{n} \mid \phi\right\rangle=\sum_{\omega} v(\omega)\left\langle\psi_{j} \mid \omega_{j}\right\rangle\left\langle\psi_{n+1} \mid \omega_{n+1}\right\rangle \prod_{k \neq j}\left\langle\psi_{k} \mid \omega_{k}\right\rangle \\
=\frac{1}{2} \sum_{\omega_{\bar{j}}}\left(v\left(\omega \mid \omega_{j}=\omega_{n+1}=s, \omega_{\bar{j}}\right)\right. \\
+v\left(\omega \mid \omega_{j}=\omega_{n+1}=t, \omega_{\bar{j}}\right) \\
+v\left(\omega \mid \omega_{j}=s, \omega_{n+1}=t, \omega_{\bar{j}}\right) \\
\left.+v\left(\omega \mid \omega_{j}=t, \omega_{n+1}=s, \omega_{\bar{j}}\right)\right)\left\langle\psi_{\bar{j}} \mid \omega_{\bar{j}}\right\rangle \\
=\frac{1}{2} \sum_{\omega_{\bar{j}}}\left(v\left(\omega \mid \omega_{j}=s, \omega_{n+1}=t, \omega_{\bar{j}}\right)\right. \\
\left.+v\left(\omega \mid \omega_{j}=t, \omega_{n+1}=s, \omega_{\bar{j}}\right)\right)\left\langle\psi_{\bar{j}} \mid \omega_{\bar{j}}\right\rangle,
\end{aligned}
$$

where $\left|\psi_{\bar{j}}\right\rangle,\left|\omega_{\bar{j}}\right\rangle$ have the same meanings as those in Eq. (15). Therefore, we have that $v(\omega)+v((j, n+1) \omega)=0$, for any $j \neq i$, where $(j, n+1) \omega$ represents the sequence obtained by exchanging the $j$ th and the $(n+1)$ th elements in $\omega=\omega_{1} \omega_{2} \ldots \omega_{n+1}$. Because $(j, k)=(j, n+$ $1)(k, n+1)(j, n+1)$, it is derived that

$$
v(\omega)+v((j, k) \omega)=0,
$$

for any $j, k$ different from $i$.

To proceed, we partition the total input system into two subsystems, the first one is the $i$ th program register, and the second one include the rest $n-1$ program registers and the data register. We use $i$ and $i$ to denote these subsystems respectively, then

$$
\begin{aligned}
|\phi\rangle & =\sum_{\omega} v(\omega)\left|\omega_{i}\right\rangle_{i}\left|\omega^{\prime}\right\rangle_{\bar{i}} \\
& =\sum_{s=1}^{m}|s\rangle_{i} \sum_{\omega^{\prime}} v\left(\omega \mid \omega_{i}=s, \omega^{\prime}\right)\left|\omega^{\prime}\right\rangle_{\bar{i}} \\
& =\sum_{s=1}^{m}|s\rangle_{i}\left|\phi_{s}^{\prime}\right\rangle_{\bar{i}},
\end{aligned}
$$

where

$$
\left|\omega^{\prime}\right\rangle=\left|\omega_{1}\right\rangle\left|\omega_{2}\right\rangle \cdots\left|\omega_{i-1}\right\rangle\left|\omega_{i+1}\right\rangle \cdots\left|\omega_{n}\right\rangle\left|\omega_{n+1}\right\rangle,
$$

and

$$
\left|\phi_{s}^{\prime}\right\rangle=\sum_{\omega^{\prime}} v\left(\omega \mid \omega_{i}=s, \omega^{\prime}\right)\left|\omega^{\prime}\right\rangle .
$$

The support space of $\operatorname{Tr}_{i}(|\phi\rangle\langle\phi|)$ is the span space of $\left|\phi_{s}^{\prime}\right\rangle$, for $1 \leq s \leq m$. From Eq. (19),

$$
\left\langle\omega^{\prime} \mid \phi_{s}^{\prime}\right\rangle=-\left\langle(j, k) \omega^{\prime} \mid \phi_{s}^{\prime}\right\rangle,
$$

where $j \neq k$. Hence, for any $\sigma \in S(n)$,

$$
\sigma\left|\phi_{s}^{\prime}\right\rangle=\operatorname{sgn}(\sigma)\left|\phi_{s}^{\prime}\right\rangle \text {. }
$$


which means that $\left|\phi_{s}^{\prime}\right\rangle$ is in $\wedge^{n} H$, for any $1 \leq s \leq m$. Then, Eq. (12) is satisfied, and the support space of $\operatorname{Tr}_{i}\left(\Pi_{i}\right)$ is in $\wedge^{n} H$.

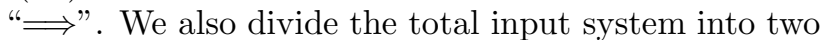
subsystems: the $i$ th program register labeled by $i$, and the rest program registers and the data register labeled by $\bar{i}$. When $j \neq i$, the total input state

$$
\left|\psi_{j}^{n}\right\rangle=\left|\psi_{i}\right\rangle_{i}\left|\psi^{\prime}\right\rangle_{\bar{i}}
$$

where

$$
\left|\psi^{\prime}\right\rangle=\left|\psi_{1}\right\rangle\left|\psi_{2}\right\rangle \cdots\left|\psi_{i-1}\right\rangle\left|\psi_{i+1}\right\rangle \cdots\left|\psi_{n}\right\rangle\left|\psi_{j}\right\rangle .
$$

Because there are two $\left|\psi_{j}\right\rangle \mathrm{s}$ in the sequence $\left|\psi_{1}\right\rangle,\left|\psi_{2}\right\rangle, \cdots$, $\left|\psi_{i-1}\right\rangle,\left|\psi_{i+1}\right\rangle, \cdots,\left|\psi_{n}\right\rangle,\left|\psi_{j}\right\rangle$, the states in this sequence are linearly dependent, from Eq.(4),

$$
\left\langle\psi^{\prime}|\Phi(n)| \psi^{\prime}\right\rangle=0
$$

From Lemma 1

$$
\left\langle\psi_{j}^{n}\left|\Pi_{i}\right| \psi_{j}^{n}\right\rangle \leq\left\langle\psi_{i}\left|\operatorname{Tr}_{i}\left(\Pi_{i}\right)\right| \psi_{i}\right\rangle\left\langle\psi^{\prime}\left|\operatorname{Tr}_{i}\left(\Pi_{i}\right)\right| \psi^{\prime}\right\rangle / \operatorname{Tr}\left(\Pi_{i}\right) .
$$

Since $\operatorname{Tr}_{i}\left(\Pi_{i}\right) \leq \wedge^{n} H$, from Eq.(27),

$$
\left\langle\psi_{j}^{n}\left|\Pi_{i}\right| \psi_{j}^{n}\right\rangle=0,
$$

for any $j \neq i$, note that $\Pi_{i}$ is a positive operator. Therefore, $\left\{\Pi_{0}, \Pi_{i}, \cdots, \Pi_{n}\right\}$ can unambiguously discriminate an arbitrary set of states $\left\{\left|\psi_{1}\right\rangle,\left|\psi_{2}\right\rangle, \cdots,\left|\psi_{n}\right\rangle\right\}$, by the quantum program $\left|\psi_{1}\right\rangle\left|\psi_{2}\right\rangle \cdots\left|\psi_{n}\right\rangle$.

The term "unambiguous" used here is in a generalized sense. When a discriminator is claimed to be unambiguous, it only means that the discriminator never makes an error, however, it may always give an inconclusive answer. For example, when $m>n$, consider a measurement $\left\{\Pi_{0}, \cdots, \Pi_{n}\right\}$, such that for any $i \neq 0, \Pi_{i}=\frac{1}{n} \Phi(n+1)$, where $\Phi(n+1)$ is the projector of $\wedge^{(n+1)} H$. In this measurement

$$
\left\langle\psi_{j}^{n}\left|\Pi_{i}\right| \psi_{j}^{n}\right\rangle=0
$$

for any $i, j$, where $i \neq 0$. Hence, it is an unambiguous programmable discriminator, however, the success probability of identifying the state is always zero.

\section{MINIMAX STRATEGY FOR DESIGNING OPTIMAL DISCRIMINATOR}

Note that when a programmable discriminator is designed, no information about states which would be discriminated by this device is given. Thus, it is reasonable to find the optimal discriminator in a minimax approach. In this strategy, the optimal discriminator is designed to maximize the minimum success probability of discriminating one state from an arbitrary state set. For a given measurement, the discrimination efficiency would be defined as

$$
p(\vec{\Pi})=\min _{\left\{\left|\psi_{i}\right\rangle\right\}} \min _{i} p_{i}(\vec{\Pi}),
$$

where $\vec{\Pi}$ is the measurement satisfying the condition for unambiguous programmable discriminator, $\left\{\left|\psi_{i}\right\rangle\right\}$ ranges over all state sets that are linearly independent, and $p_{i}(\overrightarrow{\Pi I})$ is the success probability of identifying the $i$ th state $\left|\psi_{i}\right\rangle$, by the measurement $\vec{\Pi}$, i.e.,

$$
p_{i}(\vec{\Pi})=\left\langle\psi_{i}^{n}\left|\Pi_{i}\right| \psi_{i}^{n}\right\rangle .
$$

It is observed that unambiguous programmable discriminators for $n$ quantum states form a convex set. For any $0 \leq \lambda \leq 1$, if $\vec{\Pi}$ and $\vec{\Pi}^{\prime}$ are two POVMs satisfying the condition for unambiguous programmable discriminators, $\vec{\Xi}=\lambda \vec{\Pi}+(1-\lambda) \overrightarrow{\Pi^{\prime}}$ is also an unambiguous programmable discriminator. Furthermore, the success probability of identifying the $i$ th state from a given state set by $\rightleftarrows$,

$$
\begin{aligned}
p_{i}(\vec{\Xi}) & =\left\langle\psi_{i}^{n}\left|\left(\lambda \Pi_{i}+(1-\lambda) \Pi_{i}^{\prime}\right)\right| \psi_{i}^{n}\right\rangle \\
& =\lambda\left\langle\psi_{i}^{n}\left|\Pi_{i}\right| \psi_{i}^{n}\right\rangle+(1-\lambda)\left\langle\psi_{i}^{n}\left|\Pi_{i}^{\prime}\right| \psi_{i}^{n}\right\rangle \\
& =\lambda p_{i}(\vec{\Pi})+(1-\lambda) p_{i}\left(\vec{\Pi}^{\prime}\right),
\end{aligned}
$$

is the corresponding convex combination of the success probabilities of identifying the same state by $\vec{\Pi}$ and $\overrightarrow{\Pi^{\prime}}$. Then,

$$
\begin{aligned}
p(\vec{\Xi}) & =\min _{\left\{\left|\psi_{i}\right\rangle\right\}} \min _{i} p_{i}(\vec{\Xi}) \\
& =\min _{\left\{\left|\psi_{i}\right\rangle\right\}} \min _{i} \lambda p_{i}(\vec{\Pi})+(1-\lambda) p_{i}\left(\overrightarrow{\Pi^{\prime}}\right) \\
& \geq \lambda p(\vec{\Pi})+(1-\lambda) p\left(\vec{\Pi}^{\prime}\right) .
\end{aligned}
$$

The efficiency of unambiguous programmable discriminators is a concave function.

In the remainder of this section, we provide some properties for optimal unambiguous programmable discriminators.

Lemma 2 Suppose $\vec{\Pi}$ is the optimal unambiguous programmable discriminator for $n$ states in Hilbert space $H$, then for any unitary operator $U$ in $H$, it satisfies that

$$
U^{\otimes(n+1)} \Pi_{i}\left(U^{\dagger}\right)^{\otimes(n+1)}=\Pi_{i},
$$

for $i=0, \cdots, n$.

Proof. For any unitary matrix $U$ in the Hilbert space $H$, let $\overrightarrow{\Pi^{U}}$ be a POVM, such that

$$
\Pi_{i}^{U}=U^{\otimes(n+1)} \Pi_{i}\left(U^{\dagger}\right)^{\otimes(n+1)},
$$

for $i=0, \cdots, n$. Since $\operatorname{Tr}_{i}\left(\Pi_{i}^{U}\right)=U^{\otimes n} \operatorname{Tr}_{i}\left(\Pi_{i}\right)\left(U^{\dagger}\right)^{\otimes n} \leq$ $\wedge^{n} H, \quad \overrightarrow{\Pi^{U}}$ is clearly also an unambiguous programmable discriminator. For an arbitrary set of states $\left\{\left|\psi_{1}\right\rangle, \cdots,\left|\psi_{n}\right\rangle\right\}$, the success probability of discriminating them by $\vec{\Pi}$, is the same as the success probability of discriminating $\left\{U\left|\psi_{1}\right\rangle, U\left|\psi_{2}\right\rangle, \cdots, U\left|\psi_{n}\right\rangle\right\}$ by $\overrightarrow{\Pi^{U}}$. From Eq. (31), $p\left(\overrightarrow{\Pi^{U}}\right)=p(\vec{\Pi})$, for any unitary operator U. 
Consider a new measurement $\vec{\Xi}$, which is the average of all the above measurements in a unitary distribution [21], i.e.,

$$
\Xi_{i}=\int \mathrm{d} U U^{\otimes(n+1)} \Pi_{i}\left(U^{\dagger}\right)^{\otimes(n+1)},
$$

for $i=0, \cdots, n$, where $\mathrm{d} U$ is the normalized positive invariant measure of the group $U(m)$. Clearly, $\vec{\Xi}$ is an unambiguous programmable discriminator, satisfying that, for any unitary operator $U$ in $H, U^{\otimes(n+1)} \Xi_{i}\left(U^{\dagger}\right)^{\otimes(n+1)}=$ $\Xi_{i}$, for $0 \leq i \leq n$. Because the efficiency of programmable discriminators is a concave function,

$$
\begin{aligned}
p(\vec{\Xi}) & =p\left(\int \mathrm{d} U \overrightarrow{\Pi^{U}}\right) \\
& \geq \int \mathrm{d} U p\left(\overrightarrow{\Pi^{U}}\right) \\
& =p(\vec{\Pi}) .
\end{aligned}
$$

Hence, we can substitute $\vec{\Pi}$ with $\vec{\Xi}$ as the optimal discriminator.

From above lemma, it is known that the optimal unambiguous programmable discriminators satisfies that

$$
U \operatorname{Tr}_{\bar{i}}\left(\Pi_{i}\right) U^{\dagger}=\operatorname{Tr}_{\bar{i}}\left(\Pi_{i}\right),
$$

for any unitary operator $U \in H$. So, $\operatorname{Tr}_{i}\left(\Pi_{i}\right)$ would be a diagonal matrix.

Next, we provide a relationship between the operators which consist the measurement for an optimal programmable discriminator. In the total input system of an $n$-state programmable discriminator, let us denote the $n$ program registers as subsystem $P$, and the data register as subsystem $D$. Then, we have the following lemma.

Lemma 3 Suppose $\vec{\Pi}$ is the optimal unambiguous programmable discriminator for $n$ states, then for any $\sigma \in$ $S(n)$, it holds that

$$
\left(\sigma_{P}^{-1} \otimes I_{D}\right) \Pi_{i}\left(\sigma_{P} \otimes I_{D}\right)=\Pi_{\sigma_{i}}
$$

for $i=1, \cdots, n$.

Proof. For any $\sigma \in S(n)$, let $\overrightarrow{\Pi^{\sigma}}$ be a measurement, such that

$$
\Pi_{i}^{\sigma}=\left(\sigma_{P}^{-1} \otimes I_{D}\right) \Pi_{\sigma_{i}}\left(\sigma_{P} \otimes I_{D}\right),
$$

for $i \neq 0$. Then, for any $i, j \neq 0$,

$$
\begin{aligned}
& \left\langle\psi_{j}^{n}\left|\Pi_{i}^{\sigma}\right| \psi_{j}^{n}\right\rangle \\
= & \left\langle\psi_{\sigma_{1}}\left|\left\langle\psi_{\sigma_{2}}\left|\cdots\left\langle\psi_{\sigma_{n}}\left|\left\langle\psi_{j}\left|\Pi_{\sigma_{i}}\right| \psi_{\sigma_{1}}\right\rangle\right| \psi_{\sigma_{2}}\right\rangle \cdots\right| \psi_{\sigma_{n}}\right\rangle\right| \psi_{j}\right\rangle \\
= & \left\langle\tilde{\psi}_{\sigma^{-1}(j)}^{n}\left|\Pi_{\sigma_{i}}\right| \tilde{\psi}_{\sigma^{-1}(j)}^{n}\right\rangle,
\end{aligned}
$$

where $\left|\tilde{\psi}_{k}\right\rangle=|\psi\rangle_{\sigma_{k}}$, for $k=1, \cdots, n$. Clearly, $\overrightarrow{\Pi^{\sigma}}$ is also an unambiguous programmable discriminator, whose efficiency for discriminating the states $\left\{\left|\psi_{1}\right\rangle, \cdots,\left|\psi_{n}\right\rangle\right\}$ is equal to the efficiency for discriminating $\left\{\left|\psi_{\sigma_{1}}\right\rangle, \cdots,\left|\psi_{\sigma_{n}}\right\rangle\right\}$ by $\vec{\Pi}$, which means that the two measurements have the same efficiency in minimax strategy. Hence, the measurement $\vec{\Xi}$, where

$$
\Xi_{i}=\frac{1}{n !} \sum_{\sigma \in S(n)} \Pi_{i}^{\sigma}
$$

for $1 \leq i \leq n$, is an unambiguous programmable discriminator whose efficiency is no less than $\vec{\Pi}$. In addition,

$$
\left(\sigma_{P}^{-1} \otimes I_{D}\right) \Xi_{i}\left(\sigma_{P} \otimes I_{D}\right)=\Xi_{\sigma_{i}}
$$

for any $\sigma \in S(n)$. Therefore, we can substitute $\vec{\Pi}$ by $\vec{\Xi}$.

From the above two lemmas, it is easy to conclude the following result.

Corollary 1 The optimal unambiguous programmable discriminator $\vec{\Pi}$, satisfies that

$$
\operatorname{Tr}_{i}\left(\Pi_{i}\right)=c I_{i}
$$

for $i \neq 0$, where $I_{i}$ is the identity operator on the ith subsystem, and $c$ is a constant independent of $i$.

\section{WHEN THE DIMENSION OF STATE SPACE IS EQUAL TO THE NUMBER OF DISCRIMINATED STATES}

For clarity of presentation, we divide the problem of designing optimal unambiguous programmable discriminators into two cases. In this section, we consider the case that the dimension of $H$ is equal to the number of states to be discriminated. In this situation, $\wedge^{n} H$ is a one-dimensional Hilbert space. From Theorem 1 any unambiguous programmable discriminator $\vec{\Pi}$ satisfies that $\Pi_{i}=\Pi_{i}^{\prime} \otimes \Phi(n)_{\bar{i}}$, where $\Pi_{i}^{\prime}$ is a positive operator on the $i$ th subsystem, for any $i \neq 0$. Furthermore, from Corollary 1 the optimal unambiguous programmable discriminators satisfies that

$$
\Pi_{i}=c I_{i} \otimes \Phi(n)_{\bar{i}}
$$

for $i \neq 0$. Then, we give one of our main results as follows.

Theorem 2 The optimal unambiguous programmable discriminator for $n$ states in an $n$-dimensional Hilbert space $H$ would be an measurement $\left\{\Pi_{0}, \Pi_{1}, \ldots, \Pi_{n}\right\}$ on the total input space, such that for $1 \leq i \leq n$,

$$
\Pi_{i}=\frac{n}{n+1} I_{i} \otimes \Phi(n)_{\bar{i}},
$$

and

$$
\Pi_{0}=I^{\otimes(n+1)}-\sum_{i=1}^{n} \Pi_{i}
$$


where $I$ is the identity operator on $H$, and $\Phi(n)$ is the projector of $\wedge^{n} H$. The success probability of discriminating states $\left\{\left|\psi_{1}\right\rangle,\left|\psi_{2}\right\rangle, \cdots,\left|\psi_{n}\right\rangle\right\}$ is

$$
p_{i}=\frac{n}{(n+1) !} \operatorname{det}(X),
$$

for any $1 \leq i \leq n$, where $X$ is the Gram matrix of states being discriminated.

Proof. Let $\{|1\rangle,|2\rangle, \ldots,|n\rangle\}$ be an arbitrary orthonormal basis of $H$. Then $\Phi(n)=|\phi\rangle\langle\phi|$, where

$$
\begin{aligned}
|\phi\rangle & =|1\rangle \wedge|2\rangle \wedge \cdots \wedge|n\rangle \\
& =\frac{1}{\sqrt{n !}} \sum_{\sigma \in S(n)} \operatorname{sgn}(\sigma)\left|\sigma_{1}\right\rangle\left|\sigma_{2}\right\rangle \cdots\left|\sigma_{n}\right\rangle .
\end{aligned}
$$

Consequently,

$$
\begin{aligned}
& \Pi_{i}=c \sum_{k=1}^{n}|k\rangle_{i}|\phi\rangle_{\bar{i}}\left\langlek | _ { i } \left\langle\left.\phi\right|_{\bar{i}}, i \neq 0,\right.\right. \\
& \Pi_{0}=I^{\otimes(n+1)}-c \sum_{i=1, k=1}^{n, n}|k\rangle_{i}|\phi\rangle_{\bar{i}}\left\langlek | _ { i } \left\langle\left.\phi\right|_{\bar{i}} .\right.\right.
\end{aligned}
$$

Let $G$ be the Gram matrix of $\left\{|k\rangle_{i}|\phi\rangle_{\bar{i}}: 1 \leq k \leq n, 1 \leq\right.$ $i \leq n\}$, i.e., the $(k, l)$ element in the $(i, j)$ block of matrix $G$ is the inner product of $|k\rangle_{i}|\phi\rangle_{\bar{i}}$ and $|l\rangle_{j}|\phi\rangle_{\bar{j}}$. When $i=j$, we have

$$
\left\langle\left. k\right|_{i}\left\langle\left.\phi\right|_{\bar{i}} \mid l\right\rangle_{i} \mid \phi\right\rangle_{\bar{i}}=\delta_{k, l}
$$

and when $i \neq j$, it holds that

$$
\begin{aligned}
\left\langle\left. k\right|_{i}\left\langle\left.\phi\right|_{\bar{i}} \mid l\right\rangle_{j} \mid \phi\right\rangle_{\bar{j}} & =(-1)^{i-j+1} \frac{(n-1) !}{n !} \delta_{k, l} \\
& =(-1)^{i-j+1} \frac{1}{n} \delta_{k, l} .
\end{aligned}
$$

So, the $(i, j)$ block of $G$ is

$$
G_{i j}=I \delta_{i, j}+\frac{(-1)^{i-j+1}}{n} I\left(1-\delta_{i, j}\right) .
$$

Since the eigenvalues of $\sum_{i=1, k=1}^{n, n}|k\rangle_{i}|\phi\rangle_{\bar{i}}\left\langle\left. k\right|_{i}\left\langle\left.\phi\right|_{\bar{i}}\right.\right.$ are equal to the eigenvalues of $G$, to confirm $\Pi_{0} \geq 0$, the maximum value of $c$ should be the reciprocal of maximum eigenvalue of matrix $G$, which can be calculated to be $\frac{n+1}{n}$ [20]. As a result, the maximum value of $c$ should be $\frac{n}{n+1}$.

The success probability of discriminating the $i$ th state,

$$
\begin{aligned}
p_{i} & =\left\langle\psi_{i}^{n}\left|\Pi_{i}\right| \psi_{i}^{n}\right\rangle \\
& =c\left\langle\psi^{\prime}|\Phi| \psi^{\prime}\right\rangle \\
& =\frac{c}{n !} \operatorname{det}(X) .
\end{aligned}
$$

Here

$$
\left|\psi^{\prime}\right\rangle=\left|\psi_{1}\right\rangle\left|\psi_{2}\right\rangle \cdots\left|\psi_{i-1}\right\rangle\left|\psi_{i+1}\right\rangle \cdots\left|\psi_{n}\right\rangle\left|\psi_{i}\right\rangle
$$

and $X$ is the Gram matrix of $\left\{\left|\psi_{1}\right\rangle,\left|\psi_{2}\right\rangle, \cdots,\left|\psi_{n}\right\rangle\right\}$, i.e., the $(i, j)$ element of $X$,

$$
X_{i, j}=\left\langle\psi_{i} \mid \psi_{j}\right\rangle
$$

For any $n$ linearly independent quantum states, let $H$ be the span space of them, obviously the dimension of $H$ is equal to $n$. Then, we can design the optimal programmable discriminator for $n$ states in $H$ by Theorem 2 which can unambiguously discriminate the states. However, it should be noted that the programmable discriminator designed in this way is dependent on the span space of the states being discriminated. Although such a programmable discriminator has a more general utilization than the discriminator designed according to given states, it also has an undesirable restriction. An alternative way is to design the programmable discriminators in a Hilbert space which is so great that it includes all the states which would be discriminated in application.

\section{WHEN THE DIMENSION OF STATE SPACE IS GREATER THAN THE NUMBER OF DISCRIMINATED STATES}

In this section, we consider the problem of designing unambiguous programmable discriminators for $n$ states in an $m$-dimensional Hilbert space $H$, where $m>n$. In this case, the structure of optimal unambiguous programmable discriminators is not clear by now. We conjecture that they have a similar structure to that of optimal programmable discriminators in the case that $m=n$, i.e.,

$$
\Pi_{i}=c I_{i} \otimes \Phi(n)_{\bar{i}},
$$

for $i \neq 0$. Clearly, this structure satisfies the demands offered by Lemma 2 and Lemma 3 The remainder of this section is devoted to give the optimal one of discriminators satisfying Eq.(58).

Suppose $\{|1\rangle,|2\rangle, \ldots,|m\rangle\}$ is an orthonormal basis for Hilbert space $H$. Let $\Sigma_{n}$ denote the set of all strictly increasing $n$-tuples chosen from $\{1,2, \ldots, m\}$, i.e., $\varsigma=$ $\left(\varsigma_{1}, \varsigma_{2}, \ldots, \varsigma_{n}\right) \in \Sigma_{n}$ if and only if $1 \leq \varsigma_{1}<\varsigma_{2}<\cdots<$ $\varsigma_{n} \leq m$. For all $\varsigma \in \Sigma_{n}$, let

$$
\begin{aligned}
\left|\phi_{\varsigma}\right\rangle & =\left|\varsigma_{1}\right\rangle \wedge\left|\varsigma_{2}\right\rangle \wedge \cdots \wedge\left|\varsigma_{n}\right\rangle \\
& =\sum_{\sigma \in S(n)} \operatorname{sgn}(\sigma)\left|\varsigma_{\sigma_{1}}\right\rangle\left|\varsigma_{\sigma_{2}}\right\rangle \cdots\left|\varsigma_{\sigma_{n}}\right\rangle
\end{aligned}
$$

$\left|\phi_{\varsigma}\right\rangle$ s construct an orthonormal basis for $\wedge^{n} H$, i.e., $\Phi(n)=\sum_{\varsigma \in \Sigma_{n}}\left|\phi_{\varsigma}\right\rangle\left\langle\phi_{\varsigma}\right|$, and

$$
\Pi_{i}=c \sum_{1 \leq k \leq m, \varsigma \in \Sigma_{n}}|k\rangle_{i}|\varsigma\rangle_{\bar{i}}\left\langlek | _ { i } \left\langle\left.\varsigma\right|_{\bar{i}},\right.\right.
$$

for $i \neq 0$.

Analogous to the situation that $m=n$, the maximum value of $c$ is the reciprocal of maximum eigenvalue of the 
Gram matrix of $\left\{|k\rangle_{i}|\varsigma\rangle_{\bar{i}}\right\}$, where $1 \leq i \leq n, 1 \leq k \leq m$, and $\varsigma \in \Sigma_{n}$. The elements of this Gram matrix can be expressed as $\left\langle\left. k\right|_{i}\left\langle\phi_{\varsigma}|\bar{i}| l\right\rangle_{j} \mid \phi_{\tau}\right\rangle_{\bar{j}}$.

First, if $i=j$,

$$
\left\langle\left. k\right|_{i}\left\langle\phi_{\varsigma}|\bar{i}| l\right\rangle_{i} \mid \phi_{\tau}\right\rangle_{\bar{i}}=\delta_{k, l} \delta_{\varsigma, \tau} .
$$

Next, if $i \neq j, \varsigma=\tau$, and $k, l \in \varsigma$,

$$
\left\langle\left. k\right|_{i}\left\langle\phi_{\varsigma}|\bar{i}| l\right\rangle_{j} \mid \phi_{\varsigma}\right\rangle_{\bar{j}}=(-1)^{i-j+1} \frac{1}{n} \delta_{k, l} .
$$

In addition, if the condition $i \neq j$ also holds, and there exists $\xi \in \Sigma_{n+1}$, i.e, $\xi$ is an $(n+1)$-tuple chosen from $\{1,2, \ldots, m\}$, satisfying that $\xi=\{k\} \cup \varsigma=\{l\} \cup \tau$,

$$
\left\langle\left. k\right|_{i}\left\langle\phi_{\varsigma}|\bar{i}| l\right\rangle_{j} \mid \phi_{\tau}\right\rangle_{\bar{j}}=(-1)^{j-i+\xi^{-1}(k)-\xi^{-1}(l)} \frac{1}{n}\left(1-\delta_{k, l}\right),
$$

where $\xi^{-1}(k), \xi^{-1}(l)$ denote the position of $k, l$ in the strict increasing $(n+1)$-tuple $\xi$, respectively.

Finally, all other elements $\left\langle\left. k\right|_{i}\left\langle\phi_{\varsigma}|\bar{i}| l\right\rangle_{j} \mid \phi_{\tau}\right\rangle_{\bar{j}}$ in this matrix would be zero.

Therefore, the Gram matrix is

$$
G=\left(\bigoplus_{\varsigma} \Gamma_{\varsigma}\right) \bigoplus\left(\bigoplus_{\xi} \Lambda_{\xi}\right)
$$

Here $\Gamma_{\varsigma}$ is the Gram matrix of $\left\{|k\rangle_{i}\left|\phi_{\varsigma}\right\rangle_{\bar{i}}\right\}$, where $k \in$ $\varsigma, \varsigma \in \Sigma_{n} ; \Lambda_{\xi}$ is the Gram matrix of $\left\{|k\rangle_{i}\left|\phi_{\xi-\{k\}}\right\rangle_{\bar{i}}\right\}$, where $k \in \xi, \xi \in \Sigma_{n+1}$, and $\xi-\{k\}$ denotes the strictly increasing $n$-tuple comprised of the elements in $\xi$ except $k$. The maximum eigenvalue of $G$ is the greatest one of eigenvalues of $\Gamma_{\varsigma} \mathrm{s}$ and $\Lambda_{\xi} \mathrm{s}$.

The $(i, j)$ block of matrix $\Gamma_{\varsigma}$ is

$$
I \delta_{i, j}+\frac{(-1)^{i-j+1}}{n} I\left(1-\delta_{i, j}\right),
$$

and the maximum eigenvalue of $\Gamma_{\varsigma}$ is $\frac{n+1}{n}$.

The $(k, l)$ element of the $(i, j)$ block in matrix $\Lambda_{\xi}$ is

$$
\delta_{i, j} \delta_{k, l}+\frac{(-1)^{i-j+k-l}}{n}\left(1-\delta_{k, l}\right)\left(1-\delta_{i, j}\right),
$$

and the maximum eigenvalue of $\Lambda_{\xi}$ can be calculated to be $n$.

Consequently, the maximum value of $c$ should be $\frac{1}{n}$. The optimal unambiguous programmable discriminator for $n$ quantum states in a $m$-dimensional Hilbert space $H$, which has the form given in Eq. (158), is a measurement $\left\{\Pi_{0}, \Pi_{1}, \ldots, \Pi_{n}\right\}$ on the total input system, such that for $1 \leq i \leq n$,

$$
\Pi_{i}=\frac{1}{n} I_{i} \otimes \Phi(n)_{\bar{i}},
$$

and

$$
\Pi_{0}=I^{\otimes(n+1)}-\sum_{i=1}^{n} \Pi_{i},
$$

where $I$ is the identity operator on $H$, and $\Phi(n)$ is the projector on $\wedge^{n} H$. Moreover, the success probability of discriminating states $\left\{\left|\psi_{1}\right\rangle,\left|\psi_{2}\right\rangle, \cdots,\left|\psi_{n}\right\rangle\right\}$ is

$$
p=\frac{1}{n \cdot n !} \operatorname{det}(X),
$$

where $X$ is the Gram matrix of states being discriminated.

It is easy to see that the success probability of discriminating a set of states is not related to the dimension of $H$, so we can choose $H$ a great enough Hilbert space in order to include all quantum states which may be discriminated in application. Then, the unambiguous programmable discriminator given by Eq. (67) and Eq.(68) is suitable for any $n$ states under consideration.

The success probability of this discriminator turns out to be zero, if and only if the states to be discriminated are linearly dependent. As we know, the necessary and sufficient condition for a set of states to be unambiguously discriminated is that the states are linearly independent [6]. So, the states which cannot be unambiguously discriminated by our devices are also unable to be unambiguously discriminated by any other device. In this way, we can claim that our programmable discriminators are universal.

On the other hand, in the minimax strategy, if we exactly know the set of states being discriminated, the optimal success probability for unambiguously discriminating $n$ states $\left\{\left|\psi_{i}\right\rangle\right\}$ is the minimum eigenvalue of $X$, where $X$ is the Gram matrix of $\left\{\left|\psi_{i}\right\rangle\right\}[$ ], 9$]$. Let $p_{s}$ denote this optimal efficiency, and $p$ denote the efficiency of discriminating the same states with the universal unambiguous programmable discriminator. Because $\left(p_{s}\right)^{n} \leq \operatorname{det}(X) \leq p_{s}$, , it holds that

$$
\frac{1}{n \cdot n !}\left(p_{s}\right)^{n} \leq p \leq \frac{1}{n \cdot n !} p_{s}
$$

Hence, when $n$ is large, the efficiency of the universal programmable discriminator would be quite undesirable, compared to the discriminator especially designed to known states.

\section{SUMMARY}

In this paper, the problem of designing programmable discriminators for any $n$ quantum states in a given Hilbert space $H$ is addressed. First, we give a necessary and sufficient condition for judging whether a measurement is an unambiguous programmable discriminator. Then, by utilizing the minimax strategy to evaluate the efficiency of discrimination, we offer several conditions for the optimal programmable discriminators, and give the optimal programmable discriminator in the case that the span space of the states is known. Furthermore, we propose a universal programmable discriminator, which can unambiguously discriminate any $n$ states under consideration. However, whether this discriminator is optimal under the minimax strategy is still unknown. 


\section{ACKNOWLEDGEMENTS}

We would like to thank Yuan Feng, Zhengfeng Ji, Runyao Duan, Zhaohui Wei, Guoming Wang, and Jianxin
Chen for precious discussions. This work was supported by the Natural Science Foundation of China (Grants Nos. 60503001, 60321002, and 60305005).

(2002)

[14] M.Hillery, M.Ziman, V.Bužek, Phys. Rev. A 69, 042311 (2004)

[15] J.Soubusta, A.Černoch, J.Fiurášek, M.Dušek, Phys. Rev. A 69, 052321 (2004).

[16] A.Brazier, V.Bužek, P.L.Knight, Phys. Rev. A 71, 032306 (2005).

[17] G.M. D'Ariano, P.Perinotti, Phys. Rev. Lett 94, 090401 (2005).

[18] M.Dušek, V.Bužek, Phys. Rev. A 66, 022112 (2002).

[19] J.A. Bergou, M. Hillery, Phys. Review. Lett 94, 160501 (2005).

[20] J.Fiurášek, M.Dušek, Phys. Review. A 69, 032302 (2004).

[21] A.Hayashi, M.Horibe, T.Hashimoto, Phys. Review. A 73, 012328 (2006).

[22] B.He, J.A.Bergou, Phys. Lett. A, to be published

[23] R.Bhatia, Matrix Analysis(Berlin:Springer)(1991).

M.A.Nielsen, I.L.Chuang, Phys. Rev. Lett 79, 321 (1997)

[12] G.Vidal, L.Masanes, J.I.Cirac, Phys. Rev. Lett 88, 047905 (2002).

[13] J.Fiurášek, M.Dušek, R.Filip, Phys. Rev. Lett 89, 190401 